TRANSACTIONS OF THE

AMERICAN MATHEMATICAL SOCIETY

Volume 359, Number 11, November 2007, Pages 5239-5256

S 0002-9947(07)03934-7

Article electronically published on June 4, 2007

\title{
ON THE BIHARMONIC AND HARMONIC INDICES OF THE HOPF MAP
}

\author{
E. LOUBEAU AND C. ONICIUC
}

In memoriam James Eells

\begin{abstract}
Biharmonic maps are the critical points of the bienergy functional and, from this point of view, generalize harmonic maps. We consider the Hopf map $\psi: \mathbb{S}^{3} \rightarrow \mathbb{S}^{2}$ and modify it into a nonharmonic biharmonic map $\phi: \mathbb{S}^{3} \rightarrow \mathbb{S}^{3}$. We show $\phi$ to be unstable and estimate its biharmonic index and nullity. Resolving the spectrum of the vertical Laplacian associated to the Hopf map, we recover Urakawa's determination of its harmonic index and nullity.
\end{abstract}

\section{INTRODUCTION}

In order to analyse the space of smooth maps between manifolds, Eells and Sampson introduced in 7 the family of functionals:

$$
E^{k}(\phi)=\frac{1}{2} \int_{M}\left|\left(d+d^{\star}\right)^{k} \phi\right|^{2} v_{g}, \quad k \in \mathbb{N}^{\star},
$$

for maps $\phi:(M, g) \rightarrow(N, h)$.

They quickly specialized their study to $k=1$ and called the critical points of the energy $E^{1}$ harmonic maps, the corresponding Euler-Lagrange equation being the vanishing of the tension field $\tau(\phi)=$ trace $\nabla^{\phi} d \phi$. Here $\nabla^{\phi}$ denotes the connection in the pull-back bundle $\phi^{-1} T N$.

Recently, the case of the bienergy $E^{2}$ has been the subject of some scrutiny. Its critical points are called biharmonic maps and Jiang, in [11, obtained the first and second variation formulas, showing, in particular, that a map is biharmonic if and only if $\tau^{2}(\phi)=0$, where $\tau^{2}(\phi)=-J^{\phi}(\tau(\phi))$ and $J^{\phi}$ is the Jacobi operator of the second variation for the energy. As $J^{\phi}$ is linear, any harmonic map is biharmonic, so we are interested in nonharmonic biharmonic maps.

Naturally, biharmonic submanifolds have been the first centre of attention. On surfaces of revolution, Caddeo, Montaldo and Piu obtained the parametric equation of all nongeodesic biharmonic curves on the torus and Delaunay surfaces [5], nonminimal biharmonic submanifolds of $\mathbb{S}^{3}$ are classified in $[\underline{3}$, while constructions of such submanifolds on $\mathbb{S}^{n}(n \geq 4)$ are presented in [4].

Received by the editors October 9, 2004 and, in revised form, July 1, 2005.

2000 Mathematics Subject Classification. Primary 58E20, 31B30.

Key words and phrases. Harmonic and biharmonic maps, Riemannian submersions, stability.

The authors are grateful to T. Levasseur for his help with representation theory.

The second author thanks the C.N.R.S. for a grant which made possible a three-month stay at the Université de Bretagne Occidentale in Brest. 
In the presence of nonconstant sectional curvature, a parametric description of all nongeodesic biharmonic curves of the Heisenberg group is given in [6], whereas Inoguchi classified, in [10, the biharmonic Legendre curves and Hopf cylinders on a 3-dimensional Sasakian space form and Sasahara gives in [18 the explicit representation of nonminimal biharmonic Legendre surfaces in a 5-dimensional Sasakian space.

While these results demonstrate the existence of nontrivial biharmonic maps, though not in abundance, they carry little or no indication as to their global behaviour. To this effect, some effort has been directed towards the stability of biharmonic maps. As an example, the identity map $\mathbb{S}^{n} \rightarrow \mathbb{S}^{n}$ and the totally geodesic inclusion map $\mathbb{S}^{m} \rightarrow \mathbb{S}^{n}$ are biharmonic stable and their nullities were computed in [15]. In [19, the author proved that the nonminimal biharmonic Legendre surfaces in a 5-dimensional Sasakian space are unstable.

The index of the inclusion $\mathbb{S}^{n}\left(\frac{1}{\sqrt{2}}\right) \rightarrow \mathbb{S}^{n+1}$ was computed in [13, and it is exactly one. Then they were investigated the indices of biharmonic maps in the unit Euclidean sphere $\mathbb{S}^{n+1}$ obtained from minimal Riemannian immersions in $\mathbb{S}^{n}\left(\frac{1}{\sqrt{2}}\right)$. In particular, the authors showed that the index of the nonminimal biharmonic map $\mathbb{S}^{m}\left(\sqrt{\frac{m+1}{m}}\right) \rightarrow \mathbb{S}^{m+p+1}$ derived from the generalized Veronese map is at least $m+2$, when $m \leq 4$, and at least $2 m+3$, when $m>4$.

The present article pursues the same line of research for submersions, by modifying a harmonic Riemannian submersion $\psi: M \rightarrow \mathbb{S}^{n}\left(\frac{1}{\sqrt{2}}\right)$ into a nonharmonic biharmonic subimmersion $\phi: M \rightarrow \mathbb{S}^{n+1}$ which we prove to be unstable. Concentrating on the Hopf map $\psi: \mathbb{S}^{3}(\sqrt{2}) \rightarrow \mathbb{S}^{2}\left(\frac{1}{\sqrt{2}}\right)$, we first establish the spectrum of the vertical Laplacian of $\psi$ which allows us to determine the harmonic index and nullity of $\psi$, hence recovering Urakawa's result (cf. [21]). We also give a geometric characterization of ker $J^{\psi}$, and obtain an estimate of the biharmonic index and nullity of $\phi: \mathbb{S}^{3}(\sqrt{2}) \rightarrow \mathbb{S}^{3}$ : the index is at least 11 and the nullity is greater than 8.

Throughout the paper, manifolds, metrics, and maps are assumed to be smooth, and $(M, g)$ denotes a connected Riemannian manifold without boundary. We denote by $\nabla$ the Levi-Civita connection of $(M, g)$, and for the curvature tensor field $R$ we use the sign convention $R(X, Y)=\left[\nabla_{X}, \nabla_{Y}\right]-\nabla_{[X, Y]}$, while the Laplacian on sections of $\phi^{-1} T N$ is $\Delta^{\phi}=-\operatorname{trace}\left(\nabla^{\phi}\right)^{2}$.

Let

$$
\mathbb{S}^{n}\left(\frac{r}{\sqrt{2}}\right)=\mathbb{S}^{n}\left(\frac{r}{\sqrt{2}}\right) \times\left\{\frac{r}{\sqrt{2}}\right\}=\left\{p=\left(x^{1}, \ldots, x^{n+1}, \frac{r}{\sqrt{2}}\right) \mid\left(x^{1}\right)^{2}+\cdots+\left(x^{n+1}\right)^{2}=\frac{r^{2}}{2}\right\}
$$

be a hypersphere of $\mathbb{S}^{n+1}(r)$, as proved in [3] $\mathbb{S}^{n}\left(\frac{r}{\sqrt{2}}\right)$ is a nonminimal biharmonic submanifold of $\mathbb{S}^{n+1}(r)$. Indeed, let $\eta=\frac{1}{r}\left(x^{1}, \ldots, x^{n+1},-\frac{r}{\sqrt{2}}\right)$ be a unit section of the normal bundle of $\mathbb{S}^{n}\left(\frac{r}{\sqrt{2}}\right)$ in $\mathbb{S}^{n+1}(r)$. Then, the second fundamental form of $\mathbb{S}^{n}\left(\frac{r}{\sqrt{2}}\right)$ is $B(X, Y)=\nabla d \mathbf{i}(X, Y)=-\frac{1}{r}\langle X, Y\rangle \eta$, and the tension field of the inclusion $\operatorname{map} \mathbf{i}: \mathbb{S}^{n}\left(\frac{r}{\sqrt{2}}\right) \rightarrow \mathbb{S}^{n+1}(r)$ is $\tau(\mathbf{i})=-\frac{n}{r} \eta$. Besides, by direct computation, $\tau^{2}(\mathbf{i})=0$.

Theorem 1.1. Let $M$ be a compact manifold and $\psi: M \rightarrow \mathbb{S}^{n}\left(\frac{r}{\sqrt{2}}\right)$ a nonconstant map. The map $\phi=\mathbf{i} \circ \psi: M \rightarrow \mathbb{S}^{n+1}(r)$ is nonharmonic biharmonic if and only if $\psi$ is harmonic and $e(\psi)$ is constant.

Proof. The composition law gives

$$
\tau(\phi)=\tau(\psi)-\frac{2}{r} e(\psi) \eta
$$


By straightforward calculations:

$$
\begin{aligned}
\Delta^{\phi} \tau(\phi)= & \Delta^{\psi} \tau(\psi)+\frac{2}{r^{2}} e(\psi) \tau(\psi)+\frac{1}{r^{2}} d \phi\left(\theta^{\sharp}+4 \operatorname{grad} e(\psi)\right) \\
& -\frac{1}{r}\left(\frac{4}{r^{2}}(e(\psi))^{2}+2 \Delta e(\psi)-2 \operatorname{div} \theta^{\sharp}+|\tau(\psi)|^{2}\right) \eta
\end{aligned}
$$

and

$$
\operatorname{trace} R^{\mathbb{S}^{n+1}(r)}(d \phi \cdot, \tau(\phi)) d \phi \cdot=\frac{1}{r^{2}} d \phi\left(\theta^{\sharp}\right)-\frac{2}{r^{2}} e(\psi) \tau(\psi)+\frac{4}{r^{3}}(e(\psi))^{2} \eta,
$$

where $\theta$ is a 1 -form on $M$ given by $\theta(X)=\langle d \psi(X), \tau(\psi)\rangle$, and $\theta^{\sharp} \in C(T M)$ is defined by $\left\langle\theta^{\sharp}, X\right\rangle=\theta(X), X \in C(T M)$.

Replacing in the biharmonic equation we obtain:

$$
\tau^{2}(\phi)=-\Delta^{\psi} \tau(\psi)-\frac{2}{r^{2}} d \phi\left(\theta^{\sharp}+2 \operatorname{grad} e(\psi)\right)+\frac{1}{r}\left(2 \Delta e(\psi)-2 \operatorname{div} \theta^{\sharp}+|\tau(\psi)|^{2}\right) \eta .
$$

Assume that $\phi$ is biharmonic. As the $\eta$-part of $\tau^{2}(\phi)$ vanishes:

$$
2 \Delta e(\psi)-2 \operatorname{div} \theta^{\sharp}+|\tau(\psi)|^{2}=0 .
$$

By Stokes, we deduce that $\tau(\psi)=0$ which implies $\Delta e(\psi)=0$, i.e. $e(\psi)$ is constant.

The converse is immediate.

Corollary 1.2 (4, 16]). If $\psi$ is a harmonic Riemannian immersion or submersion, then $\phi$ is nonharmonic biharmonic.

We close this section with the second variation formula for biharmonic maps in $\mathbb{S}^{n}(r)$.

Theorem 1.3 (15]). Let $\phi:(M, g) \rightarrow \mathbb{S}^{n}(r)$ be a biharmonic map. The Hessian of the bienergy $E^{2}$ at $\phi$ is:

$$
H\left(E^{2}\right)_{\phi}(V, W)=\int_{M}\left\langle I^{\phi}(V), W\right\rangle v_{g},
$$

with

$$
\begin{aligned}
r^{2} I^{\phi}(V)= & r^{2} \Delta^{\phi}\left(\Delta^{\phi} V\right)+\Delta^{\phi}\left(\operatorname{trace}\langle V, d \phi \cdot\rangle d \phi \cdot-|d \phi|^{2} V\right)+2\langle d \tau(\phi), d \phi\rangle V \\
& +|\tau(\phi)|^{2} V-2 \operatorname{trace}\langle V, d \tau(\phi) \cdot\rangle d \phi \cdot-2 \operatorname{trace}\langle\tau(\phi), d V \cdot\rangle d \phi \cdot \\
& -\langle\tau(\phi), V\rangle \tau(\phi)+\operatorname{trace}\left\langle d \phi \cdot, \Delta^{\phi} V\right\rangle d \phi \cdot \\
& +\frac{1}{r^{2}} \operatorname{trace}\langle d \phi \cdot, \operatorname{trace}\langle V, d \phi \cdot\rangle d \phi \cdot\rangle d \phi \cdot \\
& -\frac{2}{r^{2}}|d \phi|^{2} \operatorname{trace}\langle d \phi \cdot, V\rangle d \phi \cdot+2\langle d V, d \phi\rangle \tau(\phi)-|d \phi|^{2} \Delta^{\phi} V+\frac{1}{r^{2}}|d \phi|^{4} V
\end{aligned}
$$

and $V, W \in C\left(\phi^{-1} T \mathbb{S}^{n}(r)\right)$.

\section{Some general properties of the Hopf map $\psi$}

Let $\psi:(M, g) \rightarrow(N, h)$ be a submersion. For $p \in M$, call $\mathcal{V}_{p}=\operatorname{ker} d \psi_{p}$ the vertical space and $\mathcal{H}_{p}$, its orthogonal complement, the horizontal space. Any $X \in T_{p} M$ splits into $X^{\mathcal{V}}+X^{\mathcal{H}}$, where $X^{\mathcal{V}} \in \mathcal{V}_{p}$ and $X^{\mathcal{H}} \in \mathcal{H}_{p}$, and a vector field $X$ is basic if horizontal, i.e. $X=X^{\mathcal{H}}$, and $\psi$-related to $Y \in C(T N)$, i.e. $d \psi_{p}(X(p))=Y(\psi(p)), \forall p \in M$. 
The map $\psi$ is a Riemannian submersion if:

$$
h(d \psi(X), d \psi(Y))=g(X, Y), \quad \forall X, Y \in \mathcal{H}_{p} .
$$

Let $\psi: \mathbb{S}^{3}(\sqrt{2}) \rightarrow \mathbb{S}^{2}\left(\frac{1}{\sqrt{2}}\right)$ be the Hopf map given by:

$$
\psi(z, w)=\frac{1}{2 \sqrt{2}}\left(2 z \bar{w},|z|^{2}-|w|^{2}\right)
$$

or

$\psi\left(x^{1}, x^{2}, x^{3}, x^{4}\right)=\frac{1}{2 \sqrt{2}}\left(2 x^{1} x^{3}+2 x^{2} x^{4}, 2 x^{2} x^{3}-2 x^{1} x^{4},\left(x^{1}\right)^{2}+\left(x^{2}\right)^{2}-\left(x^{3}\right)^{2}-\left(x^{4}\right)^{2}\right)$,

where $z=x^{1}+\mathrm{i} x^{2}$ and $w=x^{3}+\mathrm{i} x^{4}$. The map $\psi$ is a harmonic Riemannian submersion and its fibres are totally geodesic submanifolds of $\mathbb{S}^{3}(\sqrt{2})$. More precisely, $\psi^{-1}(\psi(z, w))$ is the great circle passing through $(z, w)$ and $\mathrm{i}(z, w)=(\mathrm{i} z, \mathrm{i} w)$. Remark that $\psi^{1}, \psi^{2}$ and $\psi^{3}$ are harmonic homogeneous polynomials of degree 2 on $\mathbb{R}^{4}$.

Let $x \in \mathbb{S}^{3}(\sqrt{2}), y \in \mathbb{S}^{2}\left(\frac{1}{\sqrt{2}}\right)$ and define the vector fields:

$$
\begin{gathered}
X_{1}(x)=\frac{1}{\sqrt{2}}\left(-x^{2}, x^{1},-x^{4}, x^{3}\right), \quad X_{2}(x)=\frac{1}{\sqrt{2}}\left(-x^{3}, x^{4}, x^{1},-x^{2}\right), \\
X_{3}(x)=\frac{1}{\sqrt{2}}\left(-x^{4},-x^{3}, x^{2}, x^{1}\right), \quad X_{4}(x)=\frac{1}{\sqrt{2}}\left(-x^{2}, x^{1}, x^{4},-x^{3}\right), \\
X_{5}(x)=\frac{1}{\sqrt{2}}\left(-x^{3},-x^{4}, x^{1}, x^{2}\right), \quad X_{6}(x)=\frac{1}{\sqrt{2}}\left(-x^{4}, x^{3},-x^{2}, x^{1}\right), \\
Y_{1}(y)=\sqrt{2}\left(-y^{2}, y^{1}, 0\right), \quad Y_{2}(y)=\sqrt{2}\left(y^{3}, 0,-y^{1}\right), \quad Y_{3}(y)=\sqrt{2}\left(0,-y^{3}, y^{2}\right) .
\end{gathered}
$$

Then:

- $\left\{X_{1}, \ldots, X_{6}\right\}$ is a basis of Killing vector fields on $\mathbb{S}^{3}(\sqrt{2})$,

- $\left\{Y_{1}, Y_{2}, Y_{3}\right\}$ is a basis of Killing vector fields on $\mathbb{S}^{2}\left(\frac{1}{\sqrt{2}}\right)$,

- $X_{4}$ is $\psi$-related to $Y_{1}, X_{5}$ is $\psi$-related to $Y_{2}$, and $X_{6}$ is $\psi$-related to $Y_{3}$,

- $\left\{X_{1}, X_{2}, X_{3}\right\}$ is a global orthonormal frame on $\mathbb{S}^{3}(\sqrt{2})$,

- $X_{1}=X_{1}^{\mathcal{V}}$ and $\mathcal{V} \mathbb{S}^{3}(\sqrt{2})=\operatorname{span}\left\{X_{1}\right\}$

- $X_{2}=X_{2}^{\mathcal{H}}, X_{3}=X_{3}^{\mathcal{H}}$ and $\mathcal{H} \mathbb{S}^{3}(\sqrt{2})=\operatorname{span}\left\{X_{2}, X_{3}\right\}$,

- $d \psi_{x}\left(X_{2}\right)$ and $d \psi_{x}\left(X_{3}\right)$ form an orthonormal basis of $T_{\psi(x)} \mathbb{S}^{2}\left(\frac{1}{\sqrt{2}}\right)$.

A straightforward computation yields:

Proposition 2.1. The Levi-Civita connection on $\mathbb{S}^{3}(\sqrt{2})$ is given by:

$$
\left\{\begin{array}{l}
\nabla_{X_{1}} X_{1}=0, \quad \nabla_{X_{1}} X_{2}=-\frac{1}{\sqrt{2}} X_{3}, \quad \nabla_{X_{1}} X_{3}=\frac{1}{\sqrt{2}} X_{2}, \\
\nabla_{X_{2}} X_{1}=\frac{1}{\sqrt{2}} X_{3}, \quad \nabla_{X_{2}} X_{2}=0, \quad \nabla_{X_{2}} X_{3}=-\frac{1}{\sqrt{2}} X_{1}, \\
\nabla_{X_{3}} X_{1}=-\frac{1}{\sqrt{2}} X_{2}, \quad \nabla_{X_{3}} X_{2}=\frac{1}{\sqrt{2}} X_{1}, \quad \nabla_{X_{3}} X_{3}=0,
\end{array}\right.
$$

and

$$
\left[X_{1}, X_{2}\right]=-\sqrt{2} X_{3}, \quad\left[X_{2}, X_{3}\right]=-\sqrt{2} X_{1}, \quad\left[X_{3}, X_{1}\right]=-\sqrt{2} X_{2}
$$


Recall two general properties of Killing vector fields which will be used throughout the paper:

i) Let $X$ be a Killing vector field on a compact Riemannian manifold $(M, g)$. Denote by $\left\{\varphi_{t}\right\}_{t \in \mathbb{R}}$ its flow, and consider $f \in C^{\infty}(M)$. Then, as $\varphi_{t}$ is an isometry:

$$
\int_{M} f v_{g}=\int_{M}\left(f \circ \varphi_{t}\right) v_{g}, \quad \forall t \in \mathbb{R},
$$

and deriving by $t$ produces:

$$
0=\left.\frac{d}{d t}\right|_{t=0} \int_{M}\left(f \circ \varphi_{t}\right) v_{g}=\int_{M}(X f) v_{g} .
$$

Alternatively, if $X$ is Killing, then $X f=\operatorname{div}(f X)$ and, by Stokes, $\int_{M}(X f) v_{g}=0$. In particular:

$$
\int_{M}\left(X f_{1}\right) f_{2} v_{g}=-\int_{M} f_{1}\left(X f_{2}\right) v_{g} \text { and } \int_{M}(X f) f v_{g}=0 .
$$

ii) Having an isometry in $\varphi_{t}$ implies $\Delta\left(f \circ \varphi_{t}\right)=(\Delta f) \circ \varphi_{t}$, for any $t$, and, again, deriving by $t$ means that:

$$
\Delta(X f)=X(\Delta f)
$$

so, if $\Delta f=\lambda f, \Delta(X f)=\lambda(X f)$, i.e. $X$ preserves the eigenspaces of the Laplacian.

More specific to the Hopf map is:

\section{Lemma 2.2.}

$\operatorname{dim}\left\{f \in C^{\infty}\left(\mathbb{S}^{3}(\sqrt{2})\right) \mid X_{1} f=0, \Delta f=\lambda_{2 k} f\right\}=\operatorname{dim}\left\{\tilde{f} \in C^{\infty}\left(\mathbb{S}^{2}\left(\frac{1}{\sqrt{2}}\right)\right) \mid \Delta \tilde{f}=\mu_{k} \tilde{f}\right\}$, where $\lambda_{k}=\frac{k(k+2)}{2}$ and $\mu_{l}=2 l(l+1), k, l \in \mathbb{N}$, are the eigenvalues of the Laplacians of $\mathbb{S}^{3}(\sqrt{2})$ and $\mathbb{S}^{2}\left(\frac{1}{\sqrt{2}}\right)$.

Proof. As $\psi$ is a Riemannian submersion and its fibres are totally geodesic (hence minimal) submanifolds of $\mathbb{S}^{3}(\sqrt{2})$ (see [2]):

$$
\Delta(\tilde{f} \circ \psi)=(\Delta \tilde{f}) \circ \psi, \quad \forall \tilde{f} \in C^{\infty}\left(\mathbb{S}^{2}\left(\frac{1}{\sqrt{2}}\right)\right) .
$$

Therefore

$$
T:\left\{\tilde{f} \in C^{\infty}\left(\mathbb{S}^{2}\left(\frac{1}{\sqrt{2}}\right)\right) \mid \Delta \tilde{f}=\mu_{k} \tilde{f}\right\} \rightarrow\left\{f \in C^{\infty}\left(\mathbb{S}^{3}(\sqrt{2})\right) \mid X_{1} f=0, \Delta f=\lambda_{2 k} f\right\}
$$

defined by $T(\tilde{f})=\tilde{f} \circ \psi$ is an isomorphism, and the lemma follows.

Like any Riemannian submersion, $\psi: \mathbb{S}^{3}(\sqrt{2}) \rightarrow \mathbb{S}^{2}\left(\frac{1}{\sqrt{2}}\right)$ satisfies the coarea formula (cf. $[8]$ ):

$$
\int_{\mathbb{S}^{3}(\sqrt{2})}(\tilde{f} \circ \psi) v_{g}=2 \sqrt{2} \pi \int_{\mathbb{S}^{2}\left(\frac{1}{\sqrt{2}}\right)} \tilde{f} v_{g}, \quad \forall \tilde{f} \in C^{\infty}\left(\mathbb{S}^{2}\left(\frac{1}{\sqrt{2}}\right)\right) .
$$

\section{The Vertical Laplacian}

To a Riemannian submersion $\psi:(M, g) \rightarrow(N, h)$ one associates a second-order operator by restricting functions to a fibre and, viewing it as a submanifold, consider its induced Laplacian.

Definition 3.1 ([1]). The vertical Laplacian $\Delta^{V}$ is the differential operator defined on $(M, g)$ by:

$$
\left(\Delta^{V} f\right)(p)=\left(\Delta^{F_{p}}\left(f_{\mid F_{p}}\right)\right)(p), \quad f \in C^{\infty}(M),
$$

where $\Delta^{F_{p}}$ is the Laplace operator of the fibre $F_{p}=\psi^{-1}(\psi(p))$. 
The fibres being isometric, the spectrum of $\Delta^{V}$ is discrete.

Bérard Bergery and Bourguignon proved in [1] that, when the fibres are totally geodesic, the Laplacian $\Delta$ on $M$ and the vertical Laplacian commute, implying:

Theorem 3.2 ([1]). The Hilbert space $L^{2}(M)$ admits a Hilbert basis consisting of simultaneous eigenfunctions for $\Delta$ and $\Delta^{V}$.

Denote by $\left\{c_{i}\right\}_{i \in \mathbb{N}}$ the eigenvalues of $\Delta^{V}$ (shared with the Laplacian $\Delta^{F}$ of a generic fibre $F$ ); then $c_{0}=0, c_{i}>0$ for any $i>0$, and $c_{i} \longrightarrow \infty$ when $i \longrightarrow \infty$. Note that the multiplicities are not necessarily finite.

For the Hopf map, the corresponding vertical Laplacian is $\Delta^{V} f=-X_{1} X_{1} f$. As $X_{1}$, and by consequence $\Delta^{V}$, preserves the eigenspaces of $\Delta$, to establish the spectrum of the vertical Laplacian we restrict $\Delta^{V}$ to the eigenspaces of $\Delta$, and consider the following problem:

$$
\left\{\begin{array}{l}
\Delta f=\lambda_{k} f, \\
\Delta^{V} f=c_{l} f .
\end{array}\right.
$$

We prove that $c_{l}=\frac{(k-2 l)^{2}}{2}, l \in\{0, \ldots, k\}$, with multiplicity $2(k+1)$, with the exception of $c_{k / 2}=0$, when $k$ is even, which has multiplicity $k+1$.

Denote by $\mathbf{H}^{k}$ the set of harmonic homogeneous polynomials of degree $k$ on $\mathbb{C}^{2}$ and recall that $\operatorname{dim}_{\mathbb{C}} \mathbf{H}^{k}=(k+1)^{2}$. We work in the algebra of differential operators on polynomials $R=\mathbb{C}[z, \bar{z}, w, \bar{w}]$ and introduce the linear operators:

$$
\Delta=-4\left(\partial_{z} \partial_{\bar{z}}+\partial_{w} \partial_{\bar{w}}\right), \quad \mathrm{h}=\bar{z} \partial_{\bar{z}}-z \partial_{z}+\bar{w} \partial_{\bar{w}}-w \partial_{w} .
$$

Note that $\Delta$ is the usual Laplacian on $\mathbb{C}^{2}$ and

$$
[\Delta, \mathrm{h}]=0
$$

therefore $\mathbf{H}^{k}$ is stable under the action of $\boldsymbol{h}$.

Since $\mathrm{h}=\mathrm{i} \sqrt{2} X_{1}$, we determine the spectrum of $\mathrm{h}$ restricted to $\mathbf{H}^{k}$ to solve (3.1). The strategy is to include $\mathrm{h}$ in a Lie algebra isomorphic to $\mathfrak{s l}(2, \mathbb{C})$ acting on $\mathbf{H}^{k}$ and use representation theory to deduce its spectrum.

Let:

$$
\mathrm{e}=\mathrm{i}\left(\bar{w} \partial_{z}-\bar{z} \partial_{w}\right), \quad \mathrm{f}=\mathrm{i}\left(w \partial_{\bar{z}}-z \partial_{\bar{w}}\right) .
$$

One can easily check that:

$$
[e, f]=h, \quad[h, e]=2 e, \quad[h, f]=-2 f,
$$

making $\mathfrak{g}=\mathbb{C e}+\mathbb{C f}+\mathbb{C h}$ a Lie subalgebra of End $\mathbb{\mathbb { C }} R$ isomorphic to $\mathfrak{s l}(2, \mathbb{C})$. Moreover

$$
[\Delta, \mathrm{e}]=[\Delta, \mathrm{f}]=[\Delta, \mathrm{h}]=0,
$$

so $\mathbf{H}^{k}$ is stable under the action of $\mathfrak{g}$ and we can decompose it as a $\mathfrak{g}$-module.

Recall (cf. [9]) that there exists, up to isomorphisms, a unique $\mathfrak{g}$-module of dimension $d+1$ :

$$
E(d)=\bigoplus_{l=0}^{d} \mathbb{C} \mathrm{f}^{l}(v),
$$

where $v \in E(d)$ is determined, up to a nonzero scalar, by:

$$
\mathrm{e}(v)=0, \quad \mathrm{~h}(v)=d v .
$$


This space $E(d)$ is called the simple $\mathfrak{g}$-module of highest weight $d$ and $v$ a highest weight vector. Besides, each $\mathbb{C} f^{l}(v)$ is the eigenspace of $h$ associated to the eigenvalue $d-2 l(l \in\{0, \ldots, d\})$, i.e.

$$
\mathrm{h}\left(\mathrm{f}^{l}(v)\right)=(d-2 l) \mathrm{f}^{l}(v) .
$$

For $n \in\{0, \ldots, k\}$, let $f_{n}=\bar{z}^{n} \bar{w}^{k-n}$; then:

$$
\Delta\left(f_{n}\right)=0, \quad \mathrm{e}\left(f_{n}\right)=0, \quad \mathrm{~h}\left(f_{n}\right)=k f_{n},
$$

so $f_{n} \in \mathbf{H}^{k}$ spans a simple $\mathfrak{g}$-submodule of highest weight $k$, denoted by $V(n)$, that is $V(n) \simeq E(k)$ as $\mathfrak{g}$-modules. We deduce that $W=\sum_{n=0}^{k} V(n)$ is a $\mathfrak{g}$-submodule of $\mathbf{H}^{k}$. To show that this sum is direct, and infer from $\operatorname{dim} V(n)=k+1$ that $\mathbf{H}^{k}=\bigoplus_{n=0}^{k} V(n)$, we consider the operator:

$$
\Lambda=\left(z \partial_{z}-\bar{z} \partial_{\bar{z}}\right)-\left(w \partial_{w}-\bar{w} \partial_{\bar{w}}\right)
$$

Observe that:

$$
[\Lambda, \mathrm{f}]=0, \quad \Lambda\left(f_{n}\right)=(k-2 n) f_{n}, \quad \Lambda\left(\mathrm{f}^{l}\left(f_{n}\right)\right)=\mathrm{f}^{l}\left(\Lambda\left(f_{n}\right)\right)=(k-2 n) \mathrm{f}^{l}\left(f_{n}\right) .
$$

Therefore $V(n)$ is the eigenspace of $\Lambda$ associated to the eigenvalue $(k-2 n)$, and $\mathbf{H}^{k}$ is the direct sum of the $V(n)$ 's.

This shows that the eigenvalues of $\mathrm{h}$ are $k-2 l, l \in\{0, \ldots, k\}$, with multiplicity $k+1$ and eigenspaces $\bigoplus_{n=0}^{k} \mathbb{C} f^{l}\left(f_{n}\right)$.

In conclusion, the spectrum of the vertical Laplacian $\Delta^{V}=-X_{1} X_{1}=\frac{1}{2} \mathrm{~h}^{2}$ is $\left\{\frac{(k-2 l)^{2}}{2}: l \in\{1, \ldots, k\}\right\}$ with multiplicity $2(k+1)$, with, when $k$ is even, 0 of multiplicity $(k+1)$.

\section{The haRmonic IndeX AND NUllity of $\psi$}

The second variation of a harmonic map $\psi:(M, g) \rightarrow(N, h)$ is given by the Jacobi operator $J^{\psi}$ (cf. [14, 20]):

$$
H\left(E^{1}\right)_{\psi}(V, W)=\int_{M}\left\langle J^{\psi}(V), W\right\rangle v_{g}
$$

where $V, W \in C\left(\psi^{-1} T N\right)$ and $J^{\psi}=\Delta^{\psi}+\operatorname{trace} R^{N}(d \psi), d \psi$. In the case of the Hopf map, the Jacobi operator $J^{\psi}: C\left(\psi^{-1} T \mathbb{S}^{2}\left(\frac{1}{\sqrt{2}}\right)\right) \rightarrow C\left(\psi^{-1} T \mathbb{S}^{2}\left(\frac{1}{\sqrt{2}}\right)\right)$ is:

$$
\begin{aligned}
J^{\psi}(V) & =\Delta^{\psi} V+\operatorname{trace} R^{\mathbb{S}^{2}\left(\frac{1}{\sqrt{2}}\right)}(d \psi, V) d \psi \\
& =\Delta^{\psi} V-\operatorname{Ricci}^{\mathbb{S}^{2}\left(\frac{1}{\sqrt{2}}\right)}(V) \\
& =\Delta^{\psi} V-2 V .
\end{aligned}
$$

As $\left\{X_{2}(x), X_{3}(x)\right\}$ is a basis of $\mathcal{H}_{x}, \forall x \in \mathbb{S}^{3}(\sqrt{2})$, any section $V$ can be written $f_{2} d \psi\left(X_{2}\right)+f_{3} d \psi\left(X_{3}\right)$, where $f_{2}, f_{3} \in C^{\infty}\left(\mathbb{S}^{3}(\sqrt{2})\right)$; hence

$C\left(\psi^{-1} T \mathbb{S}^{2}\left(\frac{1}{\sqrt{2}}\right)\right)=\left\{f_{2} d \psi\left(X_{2}\right) \mid f_{2} \in C^{\infty}\left(\mathbb{S}^{3}(\sqrt{2})\right)\right\} \oplus\left\{f_{3} d \psi\left(X_{3}\right) \mid f_{3} \in C^{\infty}\left(\mathbb{S}^{3}(\sqrt{2})\right)\right\}$.

This sum is orthogonal with respect to the usual scalar product on $C\left(\psi^{-1} T \mathbb{S}^{2}\left(\frac{1}{\sqrt{2}}\right)\right)$.

A simple computation results in:

Lemma 4.1. If $X \in C\left(T \mathbb{S}^{3}(\sqrt{2})\right)$, then:

$$
\Delta^{\psi}(d \psi(X))=d \psi\left(X-\operatorname{trace} \nabla^{2} X\right)-2 \operatorname{trace} \nabla^{\psi} d \psi(, \nabla X) .
$$


Using Proposition 2.1. Lemma 4.1, the features of Killing vector fields and the properties of the second fundamental form of a Riemannian submersion, we have:

Lemma 4.2. The action of $J^{\psi}$ on $C\left(\psi^{-1} T \mathbb{S}^{2}\left(\frac{1}{\sqrt{2}}\right)\right)$ is specified by:

$$
J^{\psi}\left(f_{2} d \psi\left(X_{2}\right)\right)=\left(\Delta f_{2}\right) d \psi\left(X_{2}\right)+2 \sqrt{2}\left(X_{1} f_{2}\right) d \psi\left(X_{3}\right)
$$

and

$$
J^{\psi}\left(f_{3} d \psi\left(X_{3}\right)\right)=\left(\Delta f_{3}\right) d \psi\left(X_{3}\right)-2 \sqrt{2}\left(X_{1} f_{3}\right) d \psi\left(X_{2}\right) .
$$

By Lemma 4.2 if $\Delta f_{2}=\lambda_{k} f_{2}$ and $\Delta f_{3}=\lambda_{k} f_{3}$, then:

$$
\begin{aligned}
& J^{\psi}\left(f_{2} d \psi\left(X_{2}\right)\right)=\lambda_{k} f_{2} d \psi\left(X_{2}\right)+2 \sqrt{2}\left(X_{1} f_{2}\right) d \psi\left(X_{3}\right), \\
& J^{\psi}\left(f_{3} d \psi\left(X_{3}\right)\right)=\lambda_{k} f_{3} d \psi\left(X_{3}\right)-2 \sqrt{2}\left(X_{1} f_{3}\right) d \psi\left(X_{2}\right) .
\end{aligned}
$$

Moreover, since $X_{1}$ preserves the eigenspaces of the Laplacian, $J^{\psi}$ leaves invariant the subspace

$$
S_{\lambda_{k}}^{\psi}=\left\{f_{2} d \psi\left(X_{2}\right) \mid \Delta f_{2}=\lambda_{k} f_{2}\right\} \oplus\left\{f_{3} d \psi\left(X_{3}\right) \mid \Delta f_{3}=\lambda_{k} f_{3}\right\},
$$

for any $k \in \mathbb{N}$. If $k_{1} \neq k_{2}, S_{\lambda_{k_{1}}}^{\psi}$ and $S_{\lambda_{k_{2}}}^{\psi}$ are orthogonal one to the other.

Proposition 4.3. The eigenvalues of $J^{\psi}$ restricted to $S_{\lambda_{k}}^{\psi}$ are $\lambda_{k} \pm 2 \sqrt{2 c}$, where $c$ is an eigenvalue of $\Delta^{V}$ restricted to $\left\{f \in C^{\infty}\left(\mathbb{S}^{3}(\sqrt{2})\right) \mid \Delta f=\lambda_{k} f\right\}$, and the eigensections are:

- if $c=0$ and $X_{1} f_{2}=X_{1} f_{3}=0$, then

$$
J^{\psi}\left(f_{2} d \psi\left(X_{2}\right)+f_{3} d \psi\left(X_{3}\right)\right)=\lambda_{k}\left(f_{2} d \psi\left(X_{2}\right)+f_{3} d \psi\left(X_{3}\right)\right),
$$

- if $c>0$ and $\Delta^{V} f=c f$, then

$$
J^{\psi}\left(f d \psi\left(X_{2}\right)+\frac{1}{\sqrt{c}}\left(X_{1} f\right) d \psi\left(X_{3}\right)\right)=\left(\lambda_{k}+2 \sqrt{2 c}\right)\left(f d \psi\left(X_{2}\right)+\frac{1}{\sqrt{c}}\left(X_{1} f\right) d \psi\left(X_{3}\right)\right)
$$

and

$$
J^{\psi}\left(f d \psi\left(X_{2}\right)-\frac{1}{\sqrt{c}}\left(X_{1} f\right) d \psi\left(X_{3}\right)\right)=\left(\lambda_{k}-2 \sqrt{2 c}\right)\left(f d \psi\left(X_{2}\right)-\frac{1}{\sqrt{c}}\left(X_{1} f\right) d \psi\left(X_{3}\right)\right) .
$$

The multiplicity of each eigenvalue of $J^{\psi}$ restricted to $S_{\lambda_{k}}^{\psi}$ is $2(k+1)$.

The spectrum of the vertical Laplacian (consult Section 3) means that:

If $k=0$, then $\lambda_{0}=0, c=0$ and $\lambda_{k} \pm 2 \sqrt{2 c}=0$.

If $k=1$, then $\lambda_{1}=\frac{3}{2}, c=\frac{1}{2}$ (with multiplicity 4); thus $\lambda_{k} \pm 2 \sqrt{2 c} \in\left\{-\frac{1}{2}, \frac{7}{2}\right\}$.

If $k=2$, then $\lambda_{2}=4, c=0$ or $c=2$ and $\lambda_{k} \pm 2 \sqrt{2 c} \in\{0,4,8\}$. The multiplicity of $c=0$ is 3 and the multiplicity of $c=2$ is 6 .

Notice that $c=\frac{(k-2 l)^{2}}{2} \leq \frac{k^{2}}{2}$ so, if $k>2, \lambda_{k}>2 \sqrt{2 c}$, for any $c$, and the eigenvalues of $J^{\psi}$ are positive.

Homothetic transformations do not change the spectrum of the operator $J^{\psi}$; thus, from the above analysis, we recover Urakawa's result:

Theorem 4.4 ([21]). The Hopf map $\psi: \mathbb{S}^{3} \rightarrow \mathbb{S}^{2}$ is harmonic of index 4 and nullity 8. 
An alternative proof of Theorem 4.4 is via a direct method:

As $J^{\psi}$ preserves $S_{\lambda_{k}}^{\psi}$, we find an $L^{2}$-orthonormal basis of $S_{\lambda_{k}}^{\psi}, k=0,1$ or 2 , and compute the matrix of $J^{\psi}$ in this basis. Then, for $k>2$, we show that $J^{\psi}$ restricted to $S_{\lambda_{k}}^{\psi}$ is positive definite. The index and nullity of the Hopf map will be given by the matrices of the operator $J^{\psi}$ restricted to $S_{\lambda_{k}}^{\psi}$ :

i) $k=0$. In this case $f_{2}$ and $f_{3}$ are constants, and an $L^{2}$-orthonormal basis of $S_{\lambda_{0}}^{\psi}$ is

$$
B_{\lambda_{0}}^{\psi}=\left\{\frac{1}{c} d \psi\left(X_{2}\right), \frac{1}{c} d \psi\left(X_{3}\right)\right\},
$$

where $c^{2}=\operatorname{Vol}\left(\mathbb{S}^{3}(\sqrt{2})\right)$. We have $J^{\psi}\left(d \psi\left(X_{2}\right)\right)=J^{\psi}\left(d \psi\left(X_{3}\right)\right)=0$, so $J^{\psi}$, in the basis $B_{\lambda_{0}}^{\psi}$, is the zero matrix.

ii) $k=1$. A basis of the first eigenfunctions of the Laplacian on $\mathbb{S}^{3}(\sqrt{2})$ is $\left\{f_{i}^{1}(x)=\frac{x^{i}}{a}\right\}_{i=1}^{4}$, where $2 a^{2}=\operatorname{Vol}\left(\mathbb{S}^{3}(\sqrt{2})\right)$, making

$$
B_{\lambda_{1}}^{\psi}=\left\{f_{i}^{1} d \psi\left(X_{2}\right)\right\}_{i=1}^{4} \cup\left\{f_{j}^{1} d \psi\left(X_{3}\right)\right\}_{j=1}^{4}
$$

an $L^{2}$-orthonormal basis for $S_{\lambda_{1}}^{\psi}$. The matrix of $J^{\psi}$ restricted to $S_{\lambda_{1}}^{\psi}$ is conditioned by the action of $X_{1}, X_{2}$ and $X_{3}$ on $B_{\lambda_{1}}^{\psi}$ :

$$
\begin{aligned}
& X_{1} f_{1}^{1}=-\frac{f_{2}^{1}}{\sqrt{2}}, X_{1} f_{2}^{1}=\frac{f_{1}^{1}}{\sqrt{2}}, X_{1} f_{3}^{1}=-\frac{f_{4}^{1}}{\sqrt{2}}, X_{1} f_{4}^{1}=\frac{f_{3}^{1}}{\sqrt{2}}, \\
& X_{2} f_{1}^{1}=-\frac{f_{3}^{1}}{\sqrt{2}}, X_{2} f_{2}^{1}=\frac{f_{4}^{1}}{\sqrt{2}}, X_{2} f_{3}^{1}=\frac{f_{1}^{1}}{\sqrt{2}}, X_{2} f_{4}^{1}=-\frac{f_{2}^{1}}{\sqrt{2}}, \\
& X_{3} f_{1}^{1}=-\frac{f_{4}^{1}}{\sqrt{2}}, X_{3} f_{2}^{1}=-\frac{f_{3}^{1}}{\sqrt{2}}, X_{3} f_{3}^{1}=\frac{f_{2}^{1}}{\sqrt{2}}, X_{3} f_{4}^{1}=\frac{f_{1}^{1}}{\sqrt{2}} .
\end{aligned}
$$

Calculating $J^{\psi}\left(f_{i}^{1} d \psi\left(X_{2}\right)\right)$ and $J^{\psi}\left(f_{j}^{1} d \psi\left(X_{3}\right)\right)$ yields the matrix:

$$
\left(\begin{array}{cccccccc}
\frac{3}{2} & 0 & 0 & 0 & 0 & -2 & 0 & 0 \\
0 & \frac{3}{2} & 0 & 0 & 2 & 0 & 0 & 0 \\
0 & 0 & \frac{3}{2} & 0 & 0 & 0 & 0 & -2 \\
0 & 0 & 0 & \frac{3}{2} & 0 & 0 & 2 & 0 \\
0 & 2 & 0 & 0 & \frac{3}{2} & 0 & 0 & 0 \\
-2 & 0 & 0 & 0 & 0 & \frac{3}{2} & 0 & 0 \\
0 & 0 & 0 & 2 & 0 & 0 & \frac{3}{2} & 0 \\
0 & 0 & -2 & 0 & 0 & 0 & 0 & \frac{3}{2}
\end{array}\right)
$$

Its eigenvalues are $-\frac{1}{2}$ and $\frac{7}{2}$, both of multiplicity 4 .

iii) $k=2$. Set:

$$
\begin{gathered}
f_{1}^{2}=\frac{1}{b}\left(x^{1} x^{2}+x^{3} x^{4}\right), \quad f_{2}^{2}=\frac{1}{b}\left(x^{1} x^{2}-x^{3} x^{4}\right), \quad f_{3}^{2}=\frac{1}{b}\left(x^{1} x^{3}+x^{2} x^{4}\right), \\
f_{4}^{2}=\frac{1}{b}\left(x^{1} x^{3}-x^{2} x^{4}\right), \quad f_{5}^{2}=\frac{1}{b}\left(x^{1} x^{4}+x^{2} x^{3}\right), \quad f_{6}^{2}=\frac{1}{b}\left(x^{1} x^{4}-x^{2} x^{3}\right), \\
f_{7}^{2}=\frac{1}{2 b}\left(\left(x^{1}\right)^{2}+\left(x^{2}\right)^{2}-\left(x^{3}\right)^{2}-\left(x^{4}\right)^{2}\right), \quad f_{8}^{2}=\frac{1}{2 b}\left(\left(x^{1}\right)^{2}-\left(x^{2}\right)^{2}+\left(x^{3}\right)^{2}-\left(x^{4}\right)^{2}\right), \\
f_{9}^{2}=\frac{1}{2 b}\left(\left(x^{1}\right)^{2}-\left(x^{2}\right)^{2}-\left(x^{3}\right)^{2}+\left(x^{4}\right)^{2}\right),
\end{gathered}
$$


where $3 b^{2}=\operatorname{Vol}\left(\mathbb{S}^{3}(\sqrt{2})\right)$. Then $\left\{f_{i}^{2}\right\}_{i=1}^{9}$ is an $L^{2}$-orthonormal basis of $\{f \in$ $\left.C^{\infty}\left(\mathbb{S}^{3}(\sqrt{2})\right) \mid \Delta f=\lambda_{2} f\right\}$, and

$$
B_{\lambda_{2}}^{\psi}=\left\{f_{i}^{2} d \psi\left(X_{2}\right)\right\}_{i=1}^{9} \cup\left\{f_{j}^{2} d \psi\left(X_{3}\right)\right\}_{j=1}^{9}
$$

an $L^{2}$-orthonormal basis for $S_{\lambda_{2}}^{\psi}$. The vector fields $X_{1}, X_{2}, X_{3}$ act upon $B_{\lambda_{2}}^{\psi}$ by:

$$
\begin{gathered}
\left\{\begin{array}{l}
X_{1} f_{1}^{2}=\sqrt{2} f_{8}^{2}, X_{1} f_{2}^{2}=\sqrt{2} f_{9}^{2}, X_{1} f_{3}^{2}=0, X_{1} f_{4}^{2}=-\sqrt{2} f_{5}^{2}, X_{1} f_{5}^{2}=\sqrt{2} f_{4}^{2}, \\
X_{1} f_{6}^{2}=0, X_{1} f_{7}^{2}=0, X_{1} f_{8}^{2}=-\sqrt{2} f_{1}^{2}, X_{1} f_{9}^{2}=-\sqrt{2} f_{2}^{2},
\end{array}\right. \\
\left\{\begin{array}{c}
X_{2} f_{1}^{2}=\sqrt{2} f_{6}^{2}, X_{2} f_{2}^{2}=0, X_{2} f_{3}^{2}=\sqrt{2} f_{9}^{2}, X_{2} f_{4}^{2}=\sqrt{2} f_{7}^{2}, X_{2} f_{5}^{2}=0 \\
X_{2} f_{6}^{2}=-\sqrt{2} f_{1}^{2}, X_{2} f_{7}^{2}=-\sqrt{2} f_{4}^{2}, X_{2} f_{8}^{2}=0, X_{2} f_{9}^{2}=-\sqrt{2} f_{3}^{2},
\end{array}\right. \\
\left\{\begin{array}{l}
X_{3} f_{1}^{2}=0, X_{3} f_{2}^{2}=-\sqrt{2} f_{3}^{2}, X_{3} f_{3}^{2}=\sqrt{2} f_{2}^{2}, X_{3} f_{4}^{2}=0, X_{3} f_{5}^{2}=\sqrt{2} f_{7}^{2}, \\
X_{3} f_{6}^{2}=\sqrt{2} f_{8}^{2}, X_{3} f_{7}^{2}=-\sqrt{2} f_{5}^{2}, X_{3} f_{8}^{2}=-\sqrt{2} f_{6}^{2}, X_{3} f_{9}^{2}=0 .
\end{array}\right.
\end{gathered}
$$

From these relations, $J^{\psi}$ clearly preserves the subspaces generated by:

$$
\begin{gathered}
B_{1}=\left\{f_{1}^{2} d \psi\left(X_{2}\right), f_{6}^{2} d \psi\left(X_{2}\right), f_{8}^{2} d \psi\left(X_{2}\right)\right\} \cup\left\{f_{1}^{2} d \psi\left(X_{3}\right), f_{6}^{2} d \psi\left(X_{3}\right), f_{8}^{2} d \psi\left(X_{3}\right)\right\}, \\
B_{2}=\left\{-f_{9}^{2} d \psi\left(X_{2}\right), f_{3}^{2} d \psi\left(X_{2}\right), f_{2}^{2} d \psi\left(X_{2}\right)\right\} \cup\left\{-f_{9}^{2} d \psi\left(X_{3}\right), f_{3}^{2} d \psi\left(X_{3}\right), f_{2}^{2} d \psi\left(X_{3}\right)\right\},
\end{gathered}
$$

and

$$
B_{3}=\left\{f_{4}^{2} d \psi\left(X_{2}\right), f_{7}^{2} d \psi\left(X_{2}\right),-f_{5}^{2} d \psi\left(X_{2}\right)\right\} \cup\left\{f_{4}^{2} d \psi\left(X_{3}\right), f_{7}^{2} d \psi\left(X_{3}\right),-f_{5}^{2} d \psi\left(X_{3}\right)\right\},
$$

and is represented by the same matrix in any of these three bases:

$$
\left(\begin{array}{cccccc}
4 & 0 & 0 & 0 & 0 & 4 \\
0 & 4 & 0 & 0 & 0 & 0 \\
0 & 0 & 4 & -4 & 0 & 0 \\
0 & 0 & -4 & 4 & 0 & 0 \\
0 & 0 & 0 & 0 & 4 & 0 \\
4 & 0 & 0 & 0 & 0 & 4
\end{array}\right)
$$

of eigenvalues 0,4 and 8 , all of multiplicity 2 .

iv) For $k>2, J^{\psi}$ restricted to $S_{\lambda_{k}}^{\psi}$ is positive definite. Indeed, the equality:

$$
\begin{aligned}
& \left(J^{\psi}\left(f_{2} d \psi\left(X_{2}\right)+f_{3} d \psi\left(X_{3}\right)\right), f_{2} d \psi\left(X_{2}\right)+f_{3} d \psi\left(X_{3}\right)\right) \\
& \quad=\int_{\mathbb{S}^{3}(\sqrt{2})}\left(\lambda_{k}\left(\left(f_{2}\right)^{2}+\left(f_{3}\right)^{2}\right)-4 \sqrt{2}\left(X_{1} f_{3}\right) f_{2}\right) v_{g}
\end{aligned}
$$

calls for an upper bound of $\left|\int_{\mathbb{S}^{3}(\sqrt{2})}\left(X_{1} f_{3}\right) f_{2} v_{g}\right|$.

By Theorem 3.2, consider an $L^{2}$-orthonormal basis $\left\{f_{1}^{k}, \ldots, f_{m_{\lambda_{k}}}^{k}\right\}$ of $\{f \in$ $\left.C^{\infty}\left(\mathbb{S}^{3}(\sqrt{2})\right) \mid \Delta f=\lambda_{k} f\right\}$ such that $\Delta^{V} f_{i}^{k}=c_{i} f_{i}^{k}$, for any $i \in\left\{1, \ldots, m_{\lambda_{k}}\right\}, m_{\lambda_{k}}$ being the multiplicity of $\lambda_{k}$. We have:

$$
\Delta^{V} f_{3}=\Delta^{V}\left(\sum_{i=1}^{m_{\lambda_{k}}} a^{i} f_{i}^{k}\right)=\sum_{i=1}^{m_{\lambda_{k}}} a^{i} c_{i} f_{i}^{k}, \quad a^{i} \in \mathbb{R},
$$


and

$$
\begin{aligned}
\int_{\mathbb{S}^{3}(\sqrt{2})}\left(X_{1} f_{3}\right)^{2} v_{g} & =\int_{\mathbb{S}^{3}(\sqrt{2})}\left(\Delta^{V} f_{3}\right) f_{3} v_{g} \\
& =\sum_{i, j=1}^{m_{\lambda_{k}}} \int_{\mathbb{S}^{3}(\sqrt{2})} a^{i} c_{i} a^{j} f_{i}^{k} f_{j}^{k} v_{g}=\sum_{i, j=1}^{m_{\lambda_{k}}} a^{i} c_{i} a^{j} \delta_{i j}=\sum_{i=1}^{m_{\lambda_{k}}}\left(a^{i}\right)^{2} c_{i} \\
& \leq c \sum_{i=1}^{m_{\lambda_{k}}}\left(a^{i}\right)^{2}=c \int_{\mathbb{S}^{3}(\sqrt{2})}\left(f_{3}\right)^{2} v_{g},
\end{aligned}
$$

where $c=\max \left\{c_{1}, \ldots, c_{m_{\lambda_{k}}}\right\}$. Plugging in the Cauchy inequality

$$
\left|\int_{\mathbb{S}^{3}(\sqrt{2})}\left(X_{1} f_{3}\right) f_{2} v_{g}\right| \leq\left(\int_{\mathbb{S}^{3}(\sqrt{2})}\left(X_{1} f_{3}\right)^{2} v_{g}\right)^{\frac{1}{2}}\left(\int_{\mathbb{S}^{3}(\sqrt{2})}\left(f_{2}\right)^{2} v_{g}\right)^{\frac{1}{2}}
$$

yields:

$$
\begin{aligned}
\left|\int_{\mathbb{S}^{3}(\sqrt{2})}\left(X_{1} f_{3}\right) f_{2} v_{g}\right| & \leq \sqrt{c}\left(\int_{\mathbb{S}^{3}(\sqrt{2})}\left(f_{3}\right)^{2} v_{g}\right)^{\frac{1}{2}}\left(\int_{\mathbb{S}^{3}(\sqrt{2})}\left(f_{2}\right)^{2} v_{g}\right)^{\frac{1}{2}} \\
& \leq \frac{\sqrt{c}}{2} \int_{\mathbb{S}^{3}(\sqrt{2})}\left(f_{2}\right)^{2}+\left(f_{3}\right)^{2} v_{g}
\end{aligned}
$$

and

$$
\begin{gathered}
\left(J^{\psi}\left(f_{2} d \psi\left(X_{2}\right)+f_{3} d \psi\left(X_{3}\right)\right), f_{2} d \psi\left(X_{2}\right)+f_{3} d \psi\left(X_{3}\right)\right) \\
\geq(\lambda-2 \sqrt{2 c}) \int_{\mathbb{S}^{3}(\sqrt{2})}\left(f_{2}\right)^{2}+\left(f_{3}\right)^{2} v_{g}
\end{gathered}
$$

hence $J^{\psi}$ is positive definite.

Finally, from the eigenvalues of the above matrices, we reobtain that $\psi$ has index 4 and nullity 8.

\subsection{Description of $\operatorname{ker} J^{\psi}$.}

Theorem 4.5. The Hopf map $\psi: \mathbb{S}^{3}(\sqrt{2}) \rightarrow \mathbb{S}^{2}\left(\frac{1}{\sqrt{2}}\right)$ is a harmonic map whose Jacobi operator $J^{\psi}$ is negative definite on $\left\{d \psi\left(\operatorname{grad} f_{1}\right) \mid \Delta f_{1}=\lambda_{1} f_{1}\right\} \subset C\left(\psi^{-1} T \mathbb{S}^{2}\left(\frac{1}{\sqrt{2}}\right)\right)$.

Proof. Recall that $J^{\psi}$ is negative definite on

$$
\left\{f d \psi\left(X_{2}\right)-\sqrt{2}\left(X_{1} f\right) d \psi\left(X_{3}\right) \mid \Delta f=\lambda_{1} f\right\}
$$

so $f(x)=\langle u, x\rangle, u \in \mathbb{R}^{4}$, and $f_{1}(x)=\langle v, x\rangle$, where $v=\sqrt{2}\left(-u^{3}, u^{4}, u^{1},-u^{2}\right)$, satisfies:

$$
f d \psi\left(X_{2}\right)-\sqrt{2}\left(X_{1} f\right) d \psi\left(X_{3}\right)=\left(X_{2} f_{1}\right) d \psi\left(X_{2}\right)+\left(X_{3} f_{1}\right) d \psi\left(X_{3}\right)=d \psi\left(\operatorname{grad} f_{1}\right) .
$$

Proposition 4.6. Let $X=f_{2} X_{2}+f_{3} X_{3}$ be a horizontal vector field, where $f_{2}, f_{3} \in$ $C^{\infty}\left(\mathbb{S}^{3}(\sqrt{2})\right)$. Then $X$ is basic if and only if

$$
\Delta^{V} f_{2}=2 f_{2} \quad \text { and } \quad f_{3}=-\frac{1}{\sqrt{2}}\left(X_{1} f_{2}\right) .
$$


Proof. As $d \psi\left(X_{2}\right)=b\left(f_{9}^{2}, f_{1}^{2},-f_{4}^{2}\right)$ and $d \psi\left(X_{3}\right)=b\left(f_{2}^{2},-f_{8}^{2},-f_{5}^{2}\right)$ :

$$
d \psi\left(f_{2} X_{2}+f_{3} X_{3}\right)=b\left(f_{2} f_{9}^{2}+f_{3} f_{2}^{2}, f_{2} f_{1}^{2}-f_{3} f_{8}^{2},-f_{2} f_{4}^{2}-f_{3} f_{5}^{2}\right),
$$

and $X=f_{2} X_{2}+f_{3} X_{3}$ is basic if and only if:

$$
\left\{\begin{array} { l } 
{ X _ { 1 } ( f _ { 2 } f _ { 9 } ^ { 2 } + f _ { 3 } f _ { 2 } ^ { 2 } ) = 0 , } \\
{ X _ { 1 } ( f _ { 2 } f _ { 1 } ^ { 2 } - f _ { 3 } f _ { 8 } ^ { 2 } ) = 0 , } \\
{ X _ { 1 } ( f _ { 2 } f _ { 4 } ^ { 2 } + f _ { 3 } f _ { 5 } ^ { 2 } ) = 0 }
\end{array} \Longleftrightarrow \left\{\begin{array}{l}
\left(X_{1} f_{2}\right)+\sqrt{2} f_{3}=0, \\
\left(X_{1} f_{3}\right)-\sqrt{2} f_{2}=0
\end{array}\right.\right.
$$

and this system is equivalent to $\Delta^{V} f_{2}=2 f_{2}$ and $f_{3}=-\frac{1}{\sqrt{2}}\left(X_{1} f_{2}\right)$.

This enables a characterization of ker $J^{\psi}$.

Proposition 4.7. Let $X=f_{2} X_{2}+f_{3} X_{3}, \Delta f_{2}=\lambda_{k} f_{2}$ and $\Delta f_{3}=\lambda_{k} f_{3}$. Then $d \psi(X) \in \operatorname{ker} J^{\psi}$ if and only if $k=0$ or $k=2$ and $X$ is basic.

Proof. We saw that, if $d \psi(X) \in \operatorname{ker} J^{\psi}, k$ must be 0 or 2 .

If $k=0, f_{2}$ and $f_{3}$ are constants, and if $k=2, \operatorname{ker} J_{\mid S_{\lambda_{2}}^{\psi}}^{\psi}=\left\{f d \psi\left(X_{2}\right)-\right.$ $\left.\frac{1}{\sqrt{2}}\left(X_{1} f\right) d \psi\left(X_{3}\right) \mid \Delta^{V} f=2 f\right\}$. But $X=f X_{2}-\frac{1}{\sqrt{2}}\left(X_{1} f\right) X_{3}$, where $\Delta^{V} f=2 f$ is a basic vector field.

An even more precise description is:

Theorem 4.8. The kernel of the Jacobi operator $J^{\psi}$ of the Hopf map is:

$$
\begin{aligned}
\left\{d \psi(X) \mid X \in C\left(T \mathbb{S}^{3}(\sqrt{2})\right), X \text { is Killing }\right\} & \\
& \oplus\left\{(\operatorname{grad} \tilde{f}) \circ \psi \mid \tilde{f} \in C^{\infty}\left(\mathbb{S}^{2}\left(\frac{1}{\sqrt{2}}\right)\right), \Delta \tilde{f}=\mu_{1} \tilde{f}\right\} .
\end{aligned}
$$

Proof. First note that:

$$
\left\{Y \circ \psi \mid Y \in C\left(T \mathbb{S}^{2}\left(\frac{1}{\sqrt{2}}\right)\right), Y \text { is Killing }\right\}=\operatorname{span}\left\{d \psi\left(X_{4}\right), d \psi\left(X_{5}\right), d \psi\left(X_{6}\right)\right\} \subset S_{\lambda_{2}}^{\psi},
$$

and, as for any $\tilde{f} \in C^{\infty}\left(\mathbb{S}^{2}\left(\frac{1}{\sqrt{2}}\right)\right)$ we have $(\operatorname{grad} \tilde{f}) \circ \psi=d \psi(\operatorname{grad}(\tilde{f} \circ \psi))$,

$$
\left\{(\operatorname{grad} \tilde{f}) \circ \psi \mid \tilde{f} \in C^{\infty}\left(\mathbb{S}^{2}\left(\frac{1}{\sqrt{2}}\right)\right), \Delta \tilde{f}=\mu_{1} \tilde{f}\right\}=\{d \psi(\operatorname{grad}(\tilde{f} \circ \psi))\} \subset S_{\lambda_{2}}^{\psi} .
$$

As $X_{4}^{\mathcal{H}}, X_{5}^{\mathcal{H}}, X_{6}^{\mathcal{H}}$ and $\operatorname{grad}(\tilde{f} \circ \psi)$ are basic, by Proposition 4.7 ,

$$
\left\{Y \circ \psi \mid Y \in C\left(T \mathbb{S}^{2}\left(\frac{1}{\sqrt{2}}\right)\right), Y \text { is Killing }\right\} \subset \operatorname{ker} J^{\psi}
$$

and

Since both

$$
\left\{(\operatorname{grad} \tilde{f}) \circ \psi \mid \tilde{f} \in C^{\infty}\left(\mathbb{S}^{2}\left(\frac{1}{\sqrt{2}}\right)\right), \Delta \tilde{f}=\mu_{1} \tilde{f}\right\} \subset \operatorname{ker} J^{\psi}
$$

$\left\{Y \circ \psi \mid Y \in C\left(T \mathbb{S}^{2}\left(\frac{1}{\sqrt{2}}\right)\right), Y\right.$ is Killing $\}$ and $\left\{(\operatorname{grad} \tilde{f}) \circ \psi \mid \tilde{f} \in C^{\infty}\left(\mathbb{S}^{2}\left(\frac{1}{\sqrt{2}}\right)\right), \Delta \tilde{f}=\mu_{1} \tilde{f}\right\}$

have dimension 3 and $\psi$ has nullity 8 :

$$
\begin{aligned}
\operatorname{ker} J^{\psi}= & \operatorname{span}\left\{d \psi\left(X_{2}\right), d \psi\left(X_{3}\right)\right\} \\
& \oplus\left\{Y \circ \psi \mid Y \in C\left(T \mathbb{S}^{2}\left(\frac{1}{\sqrt{2}}\right)\right), Y \text { is Killing }\right\} \\
& \oplus\left\{(\operatorname{grad} \tilde{f}) \circ \psi \mid \tilde{f} \in C^{\infty}\left(\mathbb{S}^{2}\left(\frac{1}{\sqrt{2}}\right)\right), \Delta \tilde{f}=\mu_{1} \tilde{f}\right\} .
\end{aligned}
$$


As

$$
\begin{aligned}
\left\{d \psi(X) \mid X \in C\left(T \mathbb{S}^{3}(\sqrt{2})\right), X \text { is Killing }\right\} & =\operatorname{span}\left\{d \psi\left(X_{2}\right), d \psi\left(X_{3}\right)\right\} \\
& \oplus\left\{Y \circ \psi \mid Y \in C\left(T \mathbb{S}^{2}\left(\frac{1}{\sqrt{2}}\right)\right), Y \text { is Killing }\right\},
\end{aligned}
$$

the theorem follows.

Remark 4.9. Theorem 4.8 can be proven differently:

If $X$ is Killing, then $\Delta^{\psi}(d \psi(X))=2 d \psi(X)$, and so $J^{\psi}(d \psi(X))=0$.

Furthermore, if $Y \in C\left(T \mathbb{S}^{2}\left(\frac{1}{\sqrt{2}}\right)\right)$, one computes that:

$$
J^{\psi}(Y \circ \psi)=J^{\mathbf{1}}(Y) \circ \psi=\left(\Delta_{H}(Y)-4 Y\right) \circ \psi,
$$

where $1: \mathbb{S}^{2}\left(\frac{1}{\sqrt{2}}\right) \rightarrow \mathbb{S}^{2}\left(\frac{1}{\sqrt{2}}\right)$ is the identity map. As the eigenvalues of $\Delta_{H}$ on $\mathbb{S}^{2}\left(\frac{1}{\sqrt{2}}\right)$ are $\{2(k+1)(k+2) \mid k \in \mathbb{N}\}, J^{\psi}(Y \circ \psi)=0$ if and only if $Y$ is Killing or $Y=\operatorname{grad} \tilde{f}$, where $\tilde{f} \in C^{\infty}\left(\mathbb{S}^{2}\left(\frac{1}{\sqrt{2}}\right)\right)$ and $\Delta \tilde{f}=\mu_{1} \tilde{f}$. Now, a simple dimension count implies Theorem 4.8 .

\section{The BIHARMOniC Index AND NUllity OF $\psi$}

By definition, any harmonic map is a minimum of the bienergy, thus biharmonic stable. To ascertain the (biharmonic) nullity of the Hopf map $\psi: \mathbb{S}^{3}(\sqrt{2}) \rightarrow \mathbb{S}^{2}\left(\frac{1}{\sqrt{2}}\right)$, we need:

$$
\begin{gathered}
\operatorname{trace}\langle V, d \psi \cdot\rangle d \psi \cdot=V, \quad|d \psi|^{2}=2, \\
\operatorname{trace}\left\langle d \psi \cdot \Delta^{\psi} V\right\rangle d \psi \cdot=\Delta^{\psi} V, \quad \operatorname{trace}\langle d \psi \cdot \operatorname{trace}\langle d \psi \cdot V\rangle d \psi \cdot\rangle d \psi \cdot=V,
\end{gathered}
$$

and, replacing in (1.1), we obtain:

$$
\begin{aligned}
I^{\psi}(V) & =\Delta^{\psi} \Delta^{\psi}(V)-4 \Delta^{\psi} V+4 V \\
& =J^{\psi}\left(J^{\psi}(V)\right) .
\end{aligned}
$$

Again, since homothetic transformations do not alter the spectrum of the operator $I^{\psi}$, we attain:

Theorem 5.1. The Hopf map $\psi: \mathbb{S}^{3} \rightarrow \mathbb{S}^{2}$ is biharmonic stable and its biharmonic and harmonic nullities coincide, i.e. equal 8.

\section{ON THE BIHARMONIC INDEX AND NULLITY OF $\phi$}

Recall that, if $\psi: M \rightarrow \mathbb{S}^{n}\left(\frac{1}{\sqrt{2}}\right)$ is a nonconstant harmonic map of constant energy density, then $\phi=\mathbf{i} \circ \psi: M \rightarrow \mathbb{S}^{n+1}$ is nonharmonic biharmonic. As to its stability we have:

Theorem 6.1. Let $M$ be a compact manifold and $\psi:(M, g) \rightarrow \mathbb{S}^{n}\left(\frac{1}{\sqrt{2}}\right)$ a nonconstant harmonic map of constant energy density. The map $\phi=\mathbf{i} \circ \psi: M \rightarrow \mathbb{S}^{n+1}$ is biharmonic unstable.

Proof. Simple considerations furnish:

$$
\begin{gathered}
\operatorname{trace}\langle\eta, d \phi \cdot\rangle d \phi \cdot=0, \quad|d \phi|^{2}=2 e(\psi), \quad\langle d \tau(\phi), d \phi\rangle \eta=-4(e(\psi))^{2} \eta \\
|\tau(\phi)|^{2}=4(e(\psi))^{2}, \quad \operatorname{trace}\langle\eta, d \tau(\phi) \cdot\rangle d \phi \cdot=0, \quad \operatorname{trace}\langle\tau(\phi), d \eta \cdot\rangle d \phi \cdot=0 \\
\langle\tau(\phi), \eta\rangle \tau(\phi)=4(e(\psi))^{2} \eta, \quad \operatorname{trace}\left\langle d \phi \cdot \Delta^{\phi} \eta\right\rangle d \phi \cdot=\left(\Delta^{\phi} \eta\right)^{\top} \\
\langle d \eta, d \phi\rangle \tau(\phi)=-4(e(\psi))^{2} \eta
\end{gathered}
$$


and, by (1.1):

$$
\left(I^{\phi}(\eta), \eta\right)=\int_{M}\left(\left|\Delta^{\phi} \eta\right|^{2}-4 e(\psi)\left\langle\Delta^{\phi} \eta, \eta\right\rangle-12(e(\psi))^{2}\right) v_{g} .
$$

Since $\Delta^{\phi} \eta=2 e(\psi) \eta,\left(I^{\phi}(\eta), \eta\right)$ is strictly negative, i.e. $\phi$ is biharmonic unstable.

For the Hopf map, we set $V=d \phi(X)$, where $X \in C\left(T \mathbb{S}^{3}(\sqrt{2})\right)$ and, to find $I^{\phi}(V)$, evaluate the terms:

$$
\begin{gathered}
\operatorname{trace}\langle V, d \phi \cdot\rangle d \phi \cdot=V, \quad|d \phi|^{2}=2, \quad\langle d \tau(\phi), d \phi\rangle V=-4 V, \quad|\tau(\phi)|^{2} V=4 V, \\
\operatorname{trace}\langle V, d \tau(\phi) \cdot\rangle d \phi \cdot=-2 V, \quad \operatorname{trace}\langle\tau(\phi), d V \cdot\rangle d \phi \cdot=2 V, \quad\langle\tau(\phi), V\rangle \tau(\phi)=0, \\
\operatorname{trace}\left\langle d \phi \cdot, \Delta^{\phi} V\right\rangle d \phi \cdot=\left(\Delta^{\phi} V\right)^{\top}, \quad\langle d V, d \phi\rangle \tau(\phi)=\operatorname{trace}\left\langle\nabla^{\psi} d \psi(X), d \psi\right\rangle \tau(\phi) .
\end{gathered}
$$

Replacing in (1.1), we reach:

$$
I^{\phi}(V)=\Delta^{\phi} \Delta^{\phi} V-3 \Delta^{\phi} V+\left(\Delta^{\phi} V\right)^{\top}-3 V+2 \operatorname{trace}\left\langle\nabla^{\psi} d \psi(X), d \psi\right\rangle \tau(\phi) .
$$

Now, $\Delta^{\phi} V$ can be written

$$
\Delta^{\phi} V=\Delta^{\psi} V+2 \operatorname{trace}\left\langle\nabla^{\psi} d \psi(X), d \psi\right\rangle \eta+V,
$$

thus, if $f_{2}, f_{3} \in C^{\infty}\left(\mathbb{S}^{3}(\sqrt{2})\right)$ with $\Delta f_{2}=\lambda_{k} f_{2}$ and $\Delta f_{3}=\lambda_{k} f_{3}$,

$$
\begin{aligned}
\Delta^{\psi}\left(d \psi\left(f_{2} X_{2}\right)\right) & =\left(\lambda_{k}+2\right) f_{2} d \phi\left(X_{2}\right)+2 \sqrt{2}\left(X_{1} f_{2}\right) d \phi\left(X_{3}\right), \\
\Delta^{\psi}\left(d \psi\left(f_{3} X_{3}\right)\right) & =\left(\lambda_{k}+2\right) f_{3} d \phi\left(X_{3}\right)-2 \sqrt{2}\left(X_{1} f_{3}\right) d \phi\left(X_{2}\right), \\
\operatorname{trace}\left\langle\nabla^{\psi} d \psi\left(f_{2} X_{2}\right), d \psi\right\rangle \eta & =\left(X_{2} f_{2}\right) \eta, \operatorname{trace}\left\langle\nabla^{\psi} d \psi\left(f_{3} X_{3}\right), d \psi\right\rangle \eta=\left(X_{3} f_{3}\right) \eta .
\end{aligned}
$$

Therefore

$$
\begin{aligned}
I^{\phi}\left(f_{2} d \phi\left(X_{2}\right)\right)= & \left(\left(\left(\lambda_{k}\right)^{2}+4 \lambda_{k}\right) f_{2}-8\left(X_{1} X_{1} f_{2}\right)-4\left(X_{2} X_{2} f_{2}\right)\right) d \phi\left(X_{2}\right) \\
& +\left(4 \sqrt{2}\left(\lambda_{k}+2\right)\left(X_{1} f_{2}\right)-4\left(X_{3} X_{2} f_{2}\right)\right) d \phi\left(X_{3}\right) \\
& +\left(4 \lambda_{k}\left(X_{2} f_{2}\right)+4 \sqrt{2}\left(X_{3} X_{1} f_{2}\right)\right) \eta \\
I^{\phi}\left(f_{3} d \phi\left(X_{3}\right)\right)= & \left(-4 \sqrt{2}\left(\lambda_{k}+2\right)\left(X_{1} f_{3}\right)-4\left(X_{2} X_{3} f_{3}\right)\right) d \phi\left(X_{2}\right) \\
& +\left(\left(\left(\lambda_{k}\right)^{2}+4 \lambda_{k}\right) f_{3}-8\left(X_{1} X_{1} f_{3}\right)-4\left(X_{3} X_{3} f_{3}\right)\right) d \phi\left(X_{3}\right) \\
& +\left(4 \lambda_{k}\left(X_{3} f_{3}\right)-4 \sqrt{2}\left(X_{2} X_{1} f_{3}\right)\right) \eta .
\end{aligned}
$$

Set $W=f \eta$, where $f \in C^{\infty}\left(\mathbb{S}^{3}(\sqrt{2})\right)$; accordingly:

$$
I^{\phi}(W)=\Delta^{\phi} \Delta^{\phi} W-4 \Delta^{\phi} W+\left(\Delta^{\phi} W\right)^{\top}-12 W+4 d \phi(\operatorname{grad} f)
$$

and

$$
\Delta^{\phi} W=(\Delta f+2 f) \eta-2\left(X_{2} f\right) d \phi\left(X_{2}\right)-2\left(X_{3} f\right) d \phi\left(X_{3}\right) .
$$

If $f \in C^{\infty}\left(\mathbb{S}^{3}(\sqrt{2})\right)$ with $\Delta f=\lambda_{k} f$ :

$$
\begin{aligned}
I^{\phi}(f \eta)= & \left(-4 \lambda_{k}\left(X_{2} f\right)+4 \sqrt{2}\left(X_{1} X_{3} f\right)\right) d \phi\left(X_{2}\right) \\
& +\left(-4 \lambda_{k}\left(X_{3} f\right)-4 \sqrt{2}\left(X_{1} X_{2} f\right)\right) d \phi\left(X_{3}\right) \\
& +\left(\left(\left(\lambda_{k}\right)^{2}-16\right) f-4\left(X_{2} X_{2} f\right)-4\left(X_{3} X_{3} f\right)\right) \eta .
\end{aligned}
$$


As $X_{1}, X_{2}$ and $X_{3}$ preserve the eigenspaces of the Laplacian, $I^{\phi}$ preserves the subspace

$$
S_{\lambda_{k}}^{\phi}=\left\{f_{2} d \phi\left(X_{2}\right) \mid \Delta f_{2}=\lambda_{k} f_{2}\right\} \oplus\left\{f_{3} d \phi\left(X_{3}\right) \mid \Delta f_{3}=\lambda_{k} f_{3}\right\} \oplus\left\{f \eta \mid \Delta f=\lambda_{k} f\right\},
$$

for any $k \in \mathbb{N}$. If $k_{1} \neq k_{2}$, then $S_{\lambda_{k_{1}}}^{\phi} \perp S_{\lambda_{k_{2}}}^{\phi}$.

Remark 6.2. From the above calculations, one can obtain a quick estimation of the index of $\phi$ by observing that:

$$
\left(I^{\phi}(f \eta), f \eta\right)=-\frac{39}{4} \int_{\mathbb{S}^{3}(\sqrt{2})} f^{2} v_{g}
$$

for $\Delta f=\lambda_{1} f\left(\lambda_{1}=\frac{3}{2}\right)$, and $\left(I^{\phi}(\eta), V\right)=0$, so the index will be at least $1+4=5$.

However the sections $d \phi(\operatorname{grad} f),\left(\Delta f=\lambda_{1} f\right)$, responsible for the unstability of harmonic maps from spheres, do not contribute to the index since:

$$
\left(I^{\phi}(d \phi(\operatorname{grad} f)), d \phi(\operatorname{grad} f)\right)=\frac{9}{4} \int_{\mathbb{S}^{3}(\sqrt{2})} f^{2} v_{g}
$$

As to the nullity, it must be at least 5 , since $\left\{d \phi(X) \mid X \in C\left(\operatorname{TS}^{3}(\sqrt{2})\right), X\right.$ is Killing $\}$ $\subset \operatorname{ker} I^{\phi}$.

In order to determine more precisely the index of $I^{\phi}$ we follow the same approach as for $J^{\psi}$ :

i) $k=0$. In this case

$$
B_{\lambda_{0}}^{\phi}=\left\{\frac{1}{c} d \phi\left(X_{2}\right), \frac{1}{c} d \phi\left(X_{3}\right), \frac{1}{c} \eta\right\}
$$

is an $L^{2}$-orthonormal basis for $S_{\lambda_{0}}^{\phi}$, where $c^{2}=\operatorname{Vol}\left(\mathbb{S}^{3}(\sqrt{2})\right)$. As $I^{\phi}\left(d \phi\left(X_{2}\right)\right)=$ $I^{\phi}\left(d \phi\left(X_{3}\right)\right)=0$ and $I^{\phi}(\eta)=-16 \eta$, the matrix of $I^{\phi}$ in $B_{\lambda_{0}}^{\phi}$ is

$$
\left(\begin{array}{ccc}
0 & 0 & 0 \\
0 & 0 & 0 \\
0 & 0 & -16
\end{array}\right) .
$$

ii) $k=1$. An $L^{2}$-orthonormal basis for $S_{\lambda_{1}}^{\phi}$ is

$$
B_{\lambda_{1}}^{\phi}=\left\{f_{i}^{1} d \phi\left(X_{2}\right)\right\}_{i=1}^{4} \cup\left\{f_{j}^{1} d \phi\left(X_{3}\right)\right\}_{j=1}^{4} \cup\left\{f_{k}^{1} \eta\right\}_{k=1}^{4} .
$$

Computing $I^{\phi}\left(f_{i}^{1} d \phi\left(X_{2}\right)\right), I^{\phi}\left(f_{j}^{1} d \phi\left(X_{3}\right)\right)$ and $I^{\phi}\left(f_{k}^{1} \eta\right), i, j, k \in\{1, \ldots, 4\}$, supplies the matrix

$$
\left(\begin{array}{cccccccccccc}
\frac{57}{4} & 0 & 0 & 0 & 0 & -12 & 0 & 0 & 0 & 0 & -\sqrt{2} & 0 \\
0 & \frac{57}{4} & 0 & 0 & 12 & 0 & 0 & 0 & 0 & 0 & 0 & \sqrt{2} \\
0 & 0 & \frac{57}{4} & 0 & 0 & 0 & 0 & -12 & \sqrt{2} & 0 & 0 & 0 \\
0 & 0 & 0 & \frac{57}{4} & 0 & 0 & 12 & 0 & 0 & -\sqrt{2} & 0 & 0 \\
0 & 12 & 0 & 0 & \frac{57}{4} & 0 & 0 & 0 & 0 & 0 & 0 & -\sqrt{2} \\
-12 & 0 & 0 & 0 & 0 & \frac{57}{4} & 0 & 0 & 0 & 0 & -\sqrt{2} & 0 \\
0 & 0 & 0 & 12 & 0 & 0 & \frac{57}{4} & 0 & 0 & \sqrt{2} & 0 & 0 \\
0 & 0 & -12 & 0 & 0 & 0 & 0 & \frac{57}{4} & \sqrt{2} & 0 & 0 & 0 \\
0 & 0 & \sqrt{2} & 0 & 0 & 0 & 0 & \sqrt{2} & -\frac{39}{4} & 0 & 0 & 0 \\
0 & 0 & 0 & -\sqrt{2} & 0 & 0 & \sqrt{2} & 0 & 0 & -\frac{39}{4} & 0 & 0 \\
-\sqrt{2} & 0 & 0 & 0 & 0 & -\sqrt{2} & 0 & 0 & 0 & 0 & -\frac{39}{4} & 0 \\
0 & \sqrt{2} & 0 & 0 & -\sqrt{2} & 0 & 0 & 0 & 0 & 0 & 0 & -\frac{39}{4}
\end{array}\right)
$$


of eigenvalues $-\frac{15}{4}+2 \sqrt{10},-\frac{15}{4}-2 \sqrt{10}$ and $\frac{105}{4}$, all three of multiplicity 4 . We note that the index of $I^{\phi}$ restricted to $S_{\lambda_{1}}^{\phi}$ is given by the subspace $\left\{f \eta \mid \Delta f=\lambda_{1} f\right\}$.

iii) $k=2 . B_{\lambda_{2}}^{\phi}=\left\{f_{i}^{2} d \phi\left(X_{2}\right)\right\}_{i=1}^{9} \cup\left\{f_{j}^{2} d \phi\left(X_{3}\right)\right\}_{j=1}^{9} \cup\left\{f_{k}^{2} \eta\right\}_{k=1}^{9}$ is an $L^{2}$-orthonormal basis of $S_{\lambda_{2}}^{\phi}$. As in the harmonic case, it is enough to compute the matrix of $I^{\phi}$ in

$$
\left\{f_{i}^{2} d \phi\left(X_{2}\right)\right\}_{i \in\{1,6,8\}} \cup\left\{f_{j}^{2} d \phi\left(X_{3}\right)\right\}_{j \in\{1,6,8\}} \cup\left\{f_{k}^{2} \eta\right\}_{k \in\{1,6,8\}} .
$$

This matrix is

$$
\left(\begin{array}{ccccccccc}
56 & 0 & 0 & 0 & 0 & 40 & 0 & 8 \sqrt{2} & 0 \\
0 & 40 & 0 & 0 & 0 & 0 & -16 \sqrt{2} & 0 & 0 \\
0 & 0 & 48 & -48 & 0 & 0 & 0 & 0 & 0 \\
0 & 0 & -48 & 48 & 0 & 0 & 0 & 0 & 0 \\
0 & 0 & 0 & 0 & 40 & 0 & 0 & 0 & 16 \sqrt{2} \\
40 & 0 & 0 & 0 & 0 & 56 & 0 & -8 \sqrt{2} & 0 \\
0 & -16 \sqrt{2} & 0 & 0 & 0 & 0 & 8 & 0 & 0 \\
8 \sqrt{2} & 0 & 0 & 0 & 0 & -8 \sqrt{2} & 0 & 16 & 0 \\
0 & 0 & 0 & 0 & 16 \sqrt{2} & 0 & 0 & 0 & 8
\end{array}\right)
$$

and its eigenvalues are $0,96,24+16 \sqrt{3}, 24-16 \sqrt{3}$, of multiplicity two, and 32 of multiplicity one.

Looking at the form of the eigenvectors, we deduce that the index of $I^{\phi}$ restricted to $S_{\lambda_{2}}^{\phi}$ is given by the subspace

$$
\left\{\frac{\sqrt{3}-1}{2} d \phi(\operatorname{grad} f)+f \eta \mid \Delta f=\lambda_{2} f \text { and } f \perp\left\{\tilde{f} \circ \psi \mid \Delta \tilde{f}=\mu_{1} \tilde{f}\right\}\right\},
$$

and the kernel of $I^{\phi}$ restricted of $S_{\lambda_{2}}^{\phi}$ is

$$
\{Y \circ \psi \mid Y \text { is Killing }\} \oplus\left\{2(\tilde{f} \circ \psi) \eta+(\operatorname{grad} \tilde{f}) \circ \psi \mid \Delta \tilde{f}=\mu_{1} \tilde{f}\right\} .
$$

iv) $k>2$. Let $V=f_{2} d \phi\left(X_{2}\right)+f_{3} d \phi\left(X_{3}\right)+f \eta$, where $\Delta f_{2}=\lambda_{k} f_{2}, \Delta f_{3}=\lambda_{k} f_{3}$, $\Delta f=\lambda_{k} f$. By a straightforward computation:

$$
\begin{aligned}
& \left(I^{\phi}(V), V\right) \\
& =\int_{\mathbb{S}^{3}(\sqrt{2})}\left(\left(\left(\lambda_{k}\right)^{2}+4 \lambda_{k}\right)\left(f_{2}\right)^{2}+\left(\left(\lambda_{k}\right)^{2}+4 \lambda_{k}\right)\left(f_{3}\right)^{2}+\left(\left(\lambda_{k}\right)^{2}-16\right) f^{2}\right. \\
& \quad+4\left(X_{2} f_{2}+X_{3} f_{3}\right)^{2}+\left(2 \sqrt{2}\left(X_{1} f_{2}\right)-2\left(X_{3} f\right)\right)^{2}+\left(2 \sqrt{2}\left(X_{1} f_{3}\right)+2\left(X_{2} f\right)\right)^{2} \\
& \left.\quad-8 \sqrt{2}\left(\lambda_{k}+2\right)\left(X_{1} f_{3}\right) f_{2}-8 \lambda_{k}\left(X_{2} f\right) f_{2}-8 \lambda_{k}\left(X_{3} f\right) f_{3}\right) v_{g} \\
& \geq \int_{\mathbb{S}^{3}(\sqrt{2})}\left(\left(\left(\lambda_{k}\right)^{2}+4 \lambda_{k}\right)\left(f_{2}\right)^{2}+\left(\left(\lambda_{k}\right)^{2}+4 \lambda_{k}\right)\left(f_{3}\right)^{2}+\left(\left(\lambda_{k}\right)^{2}-16\right) f^{2}\right. \\
& \left.\quad-8 \sqrt{2}\left(\lambda_{k}+2\right)\left(X_{1} f_{3}\right) f_{2}-8 \lambda_{k}\left(X_{2} f\right) f_{2}-8 \lambda_{k}\left(X_{3} f\right) f_{3}\right) v_{g} .
\end{aligned}
$$

As

$$
\left|\int_{\mathbb{S}^{3}(\sqrt{2})}\left(X_{i} f_{2}\right) f_{3} v_{g}\right| \leq \frac{\sqrt{c}}{2} \int_{\mathbb{S}^{3}(\sqrt{2})}\left(f_{2}\right)^{2}+\left(f_{3}\right)^{2} v_{g}
$$


we obtain

$$
\begin{aligned}
\left(I^{\phi}(V), V\right) \geq & \int_{\mathbb{S}^{3}(\sqrt{2})}\left(\left(\left(\lambda_{k}\right)^{2}+4 \lambda_{k}-4 \sqrt{2 c}\left(\lambda_{k}+2\right)-4 \lambda_{k} \sqrt{c}\right)\left(\left(f_{2}\right)^{2}+\left(f_{3}\right)^{2}\right)\right. \\
& \left.+\left(\left(\lambda_{k}\right)^{2}-8 \lambda_{k} \sqrt{c}-16\right) f^{2}\right) v_{g}
\end{aligned}
$$

Let $A=\left(\lambda_{k}\right)^{2}+4 \lambda_{k}-4 \sqrt{2 c}\left(\lambda_{k}+2\right)-4 \lambda_{k} \sqrt{c}$ and $B=\left(\lambda_{k}\right)^{2}-8 \lambda_{k} \sqrt{c}-16$; then

$$
A \geq \frac{k^{2}(k+2)^{2}}{4}+2 k(k+2)-2 k(k(k+2)+4)-\sqrt{2} k^{2}(k+2)>0, \quad \forall k \geq 12
$$

and

$$
B \geq \frac{k^{2}(k+2)^{2}}{4}-2 \sqrt{2} k^{2}(k+2)-16>0, \quad \forall k \geq 10 .
$$

Therefore, $I^{\phi}$ restricted to $S_{\lambda_{k}}^{\phi}$ is positive definite for any $k \geq 12$.

From this analysis we conclude:

Theorem 6.3. The index of the biharmonic map $\phi: \mathbb{S}^{3} \rightarrow \mathbb{S}^{3}$ is at least 11 while its nullity is bounded from below by 8 . Moreover, $I^{\phi}$ is negative definite on:

$$
\begin{aligned}
\{c \eta \mid c \in \mathbb{R}\} & \oplus\left\{f \eta \mid \Delta f=\lambda_{1} f\right\} \\
& \oplus\left\{\frac{\sqrt{3}-1}{2} d \phi(\operatorname{grad} f)+f \eta \mid \Delta f=\lambda_{2} f \text { and } f \perp\left\{\tilde{f} \circ \psi \mid \Delta \tilde{f}=\mu_{1} \tilde{f}\right\}\right\},
\end{aligned}
$$

and vanishes on:

$$
\left\{d \phi(X) \mid X \in C\left(T \mathbb{S}^{3}(\sqrt{2})\right), X \text { is Killing } \oplus \oplus\left\{2(\tilde{f} \circ \psi) \eta+(\operatorname{grad} \tilde{f}) \circ \psi \mid \Delta \tilde{f}=\mu_{1} \tilde{f}\right\} .\right.
$$

6.1. The basic biharmonic index and nullity of $\phi$. Let $S$ be the subspace of $C\left(\phi^{-1} T \mathbb{S}^{3}\right)$ defined by:

$$
S=\left\{Y \circ \psi \mid Y \in C\left(T \mathbb{S}^{2}\left(\frac{1}{\sqrt{2}}\right)\right)\right\} \oplus\left\{(\tilde{f} \circ \psi) \eta \mid \tilde{f} \in C^{\infty}\left(\mathbb{S}^{2}\left(\frac{1}{\sqrt{2}}\right)\right)\right\} .
$$

Set $V=Y \circ \psi$ and $W=f \eta$, where $f=\tilde{f} \circ \psi$. We have:

$$
\begin{gathered}
I^{\phi}(V)=\Delta^{\phi} \Delta^{\phi} V-3 \Delta^{\phi} V+\left(\Delta^{\phi} V\right)^{\top}-3 V-4((\operatorname{div} Y) \circ \psi) \eta, \\
I^{\phi}(W)=\Delta^{\phi} \Delta^{\phi} W-4 \Delta^{\phi} W+\left(\Delta^{\phi} W\right)^{\top}-12 W+4(\operatorname{grad} \tilde{f}) \circ \psi, \\
\Delta^{\phi} V=\left(Y-\operatorname{trace} \nabla^{2} Y\right) \circ \psi+2((\operatorname{div} Y) \circ \psi) \eta=\left(\Delta^{\mathrm{i}} Y\right) \circ \psi, \\
\Delta^{\phi}(f \eta)=((\Delta \tilde{f}+2 \tilde{f}) \circ \psi) \eta-2(\operatorname{grad} \tilde{f}) \circ \psi=\left(\Delta^{\mathrm{i}}(\tilde{f} \eta)\right) \circ \psi,
\end{gathered}
$$

where $\mathbf{i}: \mathbb{S}^{2}\left(\frac{1}{\sqrt{2}}\right) \rightarrow \mathbb{S}^{3}$ is the canonical inclusion. From these relations we deduce that $I^{\phi}(V)=\left(I^{\mathbf{i}}(Y)\right) \circ \psi$ and $I^{\phi}(W)=\left(I^{\mathbf{i}}(\tilde{f} \eta)\right) \circ \psi$, so $I^{\phi}$ preserves the subspace $S$, and the restriction of $I^{\phi}$ to $S, I_{\mid S}^{\phi}$, has the same spectrum as $I^{\mathrm{i}}$. Thus, using Propositions 5.1 and 5.2 in [13, we conclude:

Theorem 6.4. The index of $I_{\mid S}^{\phi}$ is 1 and its nullity is 6. Moreover, the subspace which gives the index of $I_{\mid S}^{\phi}$ is $\{c \eta \mid c \in \mathbb{R}\}$ and the subspace which gives the nullity is $\{Y \circ \psi \mid Y$ is Killing $\} \oplus\left\{2(\tilde{f} \circ \psi) \eta+(\operatorname{grad} \tilde{f}) \circ \psi \mid \Delta \tilde{f}=\mu_{1} \tilde{f}\right\}$. 


\section{REFERENCES}

1. L. Bérard Bergery, J.P. Bourguignon. Laplacians and Riemannian submersions with totally geodesic fibres. Illinois J. Math., 26 (1982), 181-200. MR0650387(84m:58153)

2. M. Berger, P. Gauduchon, E. Mazet. Le spectre d'une variété riemannienne. Lecture Notes in Math., n. 194, Springer-Verlag, 1971. MR0282313 (43:8025)

3. R. Caddeo, S. Montaldo, C. Oniciuc. Biharmonic submanifolds of $\mathbb{S}^{3}$. Int. J. Math., 12 (2001), 867-876. MR.1863283 (2002k:53123)

4. R. Caddeo, S. Montaldo, C. Oniciuc. Biharmonic submanifolds in spheres. Israel J. Math., 130 (2002), 109-123. MR.1919374 (2003c:53090)

5. R. Caddeo, S. Montaldo, P. Piu. Biharmonic curves on a surface. Rend. Mat. Appl., (7) 21 (2001), no. 1-4, 143-157. MR.1884940 (2002k:58031)

6. R. Caddeo, C. Oniciuc, P. Piu. Explicit formulas for non-geodesic biharmonic curves of the Heisenberg group. Rend. Sem. Mat. Univ. Politec. Torino 62 (2004), 265-277. MR2129448

7. J. Eells, J.H. Sampson. Harmonic mappings of Riemannian manifolds. Amer. J. Math., 86 (1964), 109-160. MR0164306 (29:1603)

8. H. Federer. Geometric measure theory. Springer-Verlag, 1969. MR0257325 (41:1976)

9. W. Fulton, J. Harris. Representation Theory. Springer-Verlag, 1991. MR.1153249 (93a:20069)

10. J. Inoguchi. Submanifolds with harmonic mean curvature in contact 3-manifolds. Colloq. Math., 100 (2004), 163-179. MR2107514 (2005h:53105)

11. G.Y. Jiang. 2-harmonic maps and their first and second variational formulas. Chinese Ann. Math. Ser. A, 7 (1986), 389-402. MR0886529 (88i:58039)

12. E. Loubeau, Y.-L. Ou. The characterization of biharmonic morphisms. Differential Geometry and its Applications (Opava, 2001), Math. Publ., 3 (2001), 31-41. MR1978760(2004b:53111)

13. E. Loubeau, C. Oniciuc. The index of biharmonic maps in spheres. Compositio Math. 141 (2005), 729-745. MR2135286

14. E. Mazet. La formule de la variation seconde de l'énergie au voisinage d'une application harmonique. J. Differential Geom., 8 (1973), 279-296. MR0336767 (49:1540)

15. C. Oniciuc. On the second variation formula for biharmonic maps to a sphere. Publ. Math. Debrecen, 61 (2002), no.3-4, 613-622. MR1943720 (2003i:58031)

16. C. Oniciuc. New examples of biharmonic maps in spheres. Colloq. Math., 97 (2003), 131-139. MR2010548 (2004i:53091)

17. T. Sasahara. Quasi-minimal Lagrangian surfaces whose mean curvature vectors are eigenvectors. Demonstratio Math. 38 (2005), 185-196. MR2123733 (2005m:53110)

18. T. Sasahara. Legendre surfaces in Sasakian space forms whose mean curvature vectors are eigenvectors. Publ. Math. Debrecen 67 (2005), 285-303. MR2162123

19. T. Sasahara. Instability of biharmonic Legendre surfaces in Sasakian space forms. preprint.

20. R.T. Smith. The second variation formula for harmonic mappings. Proc. Amer. Math. Soc., 47 (1975), 229-236. MR0375386 (51:11580)

21. H. Urakawa. Stability of harmonic maps and eigenvalues of the Laplacian. Trans. American Math. Soc., 301 (1987), 557-589. MR0882704 (88g:58046)

Département de Mathématiques, Laboratoire C.N.R.S. U.M.R. 6205, Université de Bretagne Occidentale, 6, Avenue Victor le Gorgeu, CS 93837, 29238 Brest Cedex 3, FRANCE

E-mail address: loubeau@univ-brest.fr

Faculty of Mathematics, "Al.I. CuZA" University of Iasi, Bd. Carol I, no. 11, 700506 IASI, ROMANIA

E-mail address: oniciucc@uaic.ro 